\title{
P2P, DSM, and Other Products from the Complexity Factory
}

\author{
Willy Zwaenepoel \\ EPFL
}




\section{Impact of Research}

- Not so great

- Many research ideas have lost out

- Many non-research developments won out 


\section{Impact of Research}

o Not so great

- Many research ideas have lost out

- Many non-research developments won out

- Why is that?

- We make things too complex

- Note: not: things are too complex 


\section{Impact of Research}

o Not so great

- Many research ideas have lost out

- Many non-research developments won out

- Why is that?

- We make things too complex

- Not: things are too complex

o Why?

- Publishing/reviewing pushes us to complexity 


\section{Apologjies, Caveats and Excuses}

- Talk is rather polemic in nature

... things are said a little crassly

- Now a dean - intellectual life prohibited

- "There was once a dean who was so dumb, that other deans actually started noticing it" 


\section{P2P}

- Peer-to-peer

o No (central) server

- Easier to operate, maintain, scale, make more reliable ...

- Started as an application

- Proposed as an infrastructure for a large number of applications 


\section{Research on P2P}

- Concentrated largely on DHTs

- Log(n) access

o Chord, Pastry, ...

- Applicatjons: backup, streaming, ... 


\section{The Problem with P2P}

- Very little application other than illegal file sharing 


\section{Reallity Check}

- If we have learned anything about distributed computing over the last 25 years, it is that anything distributed is harder than anything centralized 


\section{Reasons for Distribution}

- You cannot handle it in one place

- Performance - controlled replication

- Availability - controlled replication

- Geographical distribution

- Google!

- Illegality - P2P

- From Napster to Gnutella, Kazaa, ...

- "Raw" traffic numbers are high

- Much of it static

- Could be handled by conventional replication (?) 


\section{Diffficulties for P2P}

- Hard to find anything

- Hard to make anything secure

- Open invitation to attack

- Actively used by RIAA (pollution attacks)

- Hard to write anything 


\section{Advantages for P2P Research}

- Complex to find anything

- Complex to make anything secure

- Complex to write anything 


\section{Advantages for P2P Research}

- Complex to find anything

- Complex to make anything secure

- Complex to write anything

- Complexity begets papers

o P2P = Paper-to-Paper 


\section{There are Applications}

- Large fille multicast

- Can be handled by very simple techniques

- BitTorrent

- It should worry us that these come from non-research corners of the world! 


\section{DSM}

- Distributed shared memory

- Parallel computing on clusters

- Distributed memories abstracted as a single shared memory

- Easier to write programs

- Usually by page faulting

- TreadMarks (ParallelTools) 


\section{Reality Check}

- Clusters are only suitable for coarsegrained parallel computation

o A fortjori true for DSM 


\section{Problems with Fine-Grained DSM}

- Expensive synchronization

- Expensive fine-grained data sharing

- Smaller than a page

- False sharing (can be solved)

- True sharing 


\section{Advantages for DSM Research}

- Complex fine-grain synchronization

- Complex fine-grain data sharing

- Compiler, language, runtime, ...

- Complexity begets papers ... 


\section{TreadMarks}

- (Almost) every paper or grant for research on fine-grain DSM was accepted

- (Almost) every paper or grant for research on coarse-grained DSM was rejected

- It turns out that for real applications a page is not large enough! 


\section{Coarse-graijn Applications}

- Large (independent) units of computation

- Large chunks of data

- 1 page $=4 \mathrm{k}$

- Not very large at all

- Page faulting brings in one page at a time

- Message passing brings in whole data segment at a time (> page)

- Can be and was done with DSM

- Increase page size (!!)

- Compiler support 


\section{Competition is Message Passing}

- MPI (Message Passing Interface)

- Low abstraction

o No room for complexity fabrication

- As a result more successful

- It should worry us that MPI did not come from distributed systems research but from linear algebra! 


\section{Server Performance}

- At the beginning of the Internet boom, server performance was badly lagging

- Multithreaded or multijprocess servers

- Context switching

- Locking

- Two types of solutions

- Exokernel

- Event-driven servers 


\section{Event-Driven Servers}

- Events

- Incoming request, i/o completion, ...

- Single thread, event loop

- Event handler per event

- Straight code (no blocking)

- At end:

- nonblocking or asynchronous i/o

- create (hand-made) continuation 


\section{Advantages}

- No multithreading

- No context switching

- No locking (at least on uniprocessor)

- Control over order of event handlling

- Not bound by OS scheduler 


\section{Flash}

- Most popular event-driven Web server

- Combined multithreaded / event-driven

- Many follow-ons

- iMimic Networking 


\section{Realjity Check}

o lt's too complex

- Maybe Ph.D.s can figure it out

- Your average industry programmer cannot

- Actually, most Ph.D.s can't either

- Many (expensive) bugs 


\section{How the Problem was Solved}

- Linux $O(1)$ thread scheduler

- Linux futex

- User-level locking

- No overhead if no contention

- Benefits of event-driven remain

o But too small to warrant complexity 


\section{How the Problem was Solved}

- The main servers are all process-based or thread-based (Apache, MySQL)

- It should worry us that these servers did not come out of research! 


\section{Paijnful Observations (1)}

- Most of the strong research trends have not found much application

- Non-research designs have won out

- Has to do with this fabricated complexity 


\section{Paijnful Observatjons (2)}

- Has to do with publishing/reviewing

- Simple papers tend to get rejected

- Complex papers tend to get in 


\section{Your Average Review Form}

- Novelty

- Excitement

- Writing

- Confidence 


\section{Some Questions to Add?}

- Does the added functionality justify the increase in complexity?

- Does the performance improvement justify the increase in complexity?

- Could this system be maintained by an above-average programmer in industry?

- Does this paper simplify a known solution to a worthwhile problem? 


\section{Some Likely Review Comments}

๑ «ncremental »

o «ngineering »

० « Nothing new »

० « Boring » 


\section{It IS Possible}

- Virtual machines

- Provide simple solutions to real problems

- Server consolidation

- Migration 


\section{Virtual Machines}

- Virtual machine monitor

- VMM provides a number of VMs

- IBM VM

- VMWare

- Xen

- Open-source

- Paravirtualization (VM machine) 


\section{Provenance}

- DISCO: a very complex OS for SMPs

- VMWare:

- Simplified to Linux/Windows on one machine

- Precise virtualization on x86 very complex

- Xen

- Paravirtualization to improve performance and decrease complexity

- VMM less complex

- Guest OS (slightly) more complex

oPerformance better (?) 


\section{The Way of All Technology}

- All technology

- Becomes more complex on the inside

- Becomes less complex on the outside

- Example: car, Windows (?!)

- Not sure it fully applies to software

- Most complex systems ever built

- Rare example of discrete complex system

- Maybe we are over the limit already 


\section{Nonetheless}

- Success = interfaces defined early?

- Very successful systems

- Apache, MySQL, MPI, VMWare, Xen

- Interfaces stable (few iterations)

- Internal complexity grew

- Less successful systems

- DSM, event-driven

- Interfaces unstable, complexified 


\section{Standardization (!?)}

- I am afriaid some of it is necessary

o Find a way through publishing system 


\section{Other People's Advice}

- Lampson: « Keep it simple »

- True, but somewhat impractical

- Einstein: « Everything should be as simple as possible, but no more than that $»$

- Implement functionality at the right interface

- Keep interfaces stable 


\section{Lessons}

- Brute force often (not always) works

- Our publishing and reviewing system pushes us in the opposite direction 


\section{More Lessons}

- It is the interface, stupid

- The implementation can be complex

- The interface has to be simple and stable 
Thank you 